\title{
LAPLACIANS ON BIPARTITE METRIC GRAPHS
}

\author{
Pavel Kurasov and Jonathan Rohleder
}

\begin{abstract}
We study spectral properties of the standard (also called Kirchhoff) Laplacian and the anti-standard (or anti-Kirchhoff) Laplacian on a finite, compact metric graph. We show that the positive eigenvalues of these two operators coincide whenever the graph is bipartite; this leads to a precise relation between their eigenvalues enumerated with multiplicities and including the possible eigenvalue zero. Several spectral inequalities for, e.g., trees are among the consequences of this. In the second part we study inequalities between standard and Dirichlet eigenvalues in more detail and expose another connection to bipartiteness.
\end{abstract}

Mathematics subject classification (2010): 81Q35, 34B45, 34L05.

Keywords and phrases: Quantum graph, Laplacian, eigenvalues, bipartite graph, spectral inequalities.

\section{REFERENCES}

[1] R. BAnd, G. Berkolaiko And T. Weyand, Anomalous nodal count and singularities in the dispersion relation of honeycomb graphs, J. Math. Phys., 56, 2015, 122111, 20 pp.

[2] R. BAND AND G. LÉvy, Quantum graphs which optimize the spectral gap, Ann. Henri Poincaré, 18, 2017, 3269-3323.

[3] J. VON BELOW, A characteristic equation associated to an eigenvalue problem on $c^{2}$-networks, Linear Algebra Appl., 71, 1985, 309-325.

[4] J. VON BELOW AND J. A. LUBARY, Harmonic functions on metric graphs under the anti-Kirchhoff law, Results Math., 74, 2019, 74:36.

[5] J. VON BELOW AND D. MUGNOLO, The spectrum of the Hilbert space valued second derivative with general self-adjoint boundary conditions, Linear Algebra Appl., 439, 2013, 1792-1814.

[6] G. Berkolaiko, J. Kennedy, P. Kurasov and D. Mugnolo, Edge connectivity and the spectral gap of combinatorial and quantum graphs, J. Phys. A, 50, 2017, 365201.

[7] G. Berkolaiko, J. Kennedy, P. Kurasov and D. Mugnolo, Surgery principles for the spectral analysis of quantum graphs, Trans. Amer. Math. Soc., 372 (2019), no. 7, 5153-5197.

[8] G. Berkolaiko AND P. Kuchment, Introduction to quantum graphs, Mathematical Surveys and Monographs 186, American Mathematical Society, Providence, RI, 2013.

[9] F. R. K. Chung, Spectral Graph Theory, CBMS Regional Conference Series in Mathematics 92, American Mathematical Society, Providence, RI, 1997.

[10] P. EXner, Momentum operators on graphs, Proc. Sympos. Pure Math., 87, 2013, 105-118.

[11] N. FILONOv, On an inequality for the eigenvalues of the Dirichlet and Neumann problems for the Laplace operator, Algebra i Analiz, 16, 2004, 172-176.

[12] L. Friedlander, Some inequalities between Dirichlet and Neumann eigenvalues, Arch. Rational Mech. Anal., 116, 1991, 153-160.

[13] L. FRIEDLANDER, Extremal properties of eigenvalues for a metric graph, Ann. Inst. Fourier, 55, 2005, 199-211.

[14] L. FRIEDLANDER, The Dirichlet-to-Neumann operator for quantum graphs, preprint, arXiv: 1712.08223.

[15] S. A. Fulling, P. Kuchment And J. H. Wilson, Index theorems for quantum graphs, J. Phys. A, 40, 2007, 14165-14180.

[16] J. B. KENNEDY, A sharp eigenvalue bound for quantum graphs in terms of their diameter, to appear in Operator Theory: Advances and Applications, vol. 281, Birkhäuser, 2020. 
[17] J. B. Kennedy, P. Kurasov, G. Malenová and D. Mugnolo, On the spectral gap of a quantum graph, Ann. Henri Poincaré, 17, 2016, 2439-2473.

[18] P. KURASOV, Schrödinger operators on graphs and geometry. I. Essentially bounded potentials, J. Funct. Anal., 254, 2008, 934-953.

[19] P. Kurasov, G. MALENOvÁ AND S. NABOKO, Spectral gap for quantum graphs and their connectivity, J. Phys. A, 46, 2013, 275309.

[20] P. KURASOV AND S. NABOKO, Rayleigh estimates for differential operators on graphs, J. Spectral Theory, 4, 2014, 211-219.

[21] D. Mugnolo, Semigroup Methods for Evolution Equations on Networks, Springer, Cham, 2014.

[22] S. Nicaise, Spectre des résaux topologiques finis, Bull. Sci. Math., 111, 1987, 401-413.

[23] J. ZhaO AND G. SHI, Eigenvalue estimates for the Laplacian with anti-Kirchhoff conditions on a metric tree, J. Math. Anal. Appl., 477, 2019, 670-684.

[24] J. Rohleder, Eigenvalue estimates for the Laplacian on a metric tree, Proc. Amer. Math. Soc., 145, 2017, 2119-2129.

[25] J. Rohleder AND C. SEIFERT, Spectral monotonicity for Schrödinger operators on metric graphs, to appear in Operator Theory: Advances and Applications, vol. 281, Birkhäuser, 2020. 